[Agr. Biol. Chem., Vol. 31, No. 10, p. 1248 1250, 1967]

\title{
Isolation of Fumaric Acid from Silkworm Pupas as a Causative Agent to Release Reducing Sugars in Embryoless Rice Endosperms
}

Sir:

It has been known that $\alpha$-amylase activity in the endosperm of barley ${ }^{1 /}$ and rice ${ }^{21}$ is promoted by gibberellins. Recently Ogawa ${ }^{31}$ and Ogawa and Imamura ${ }^{41}$ found that animal tissue extracts resulted in the release of reducing sugars from embryoless rice endosperms. It was suggested by these authors that effect was due to the presence of gibberellin-like substances in the tissue. In this paper, however, we wish to describe the isolation of fumaric acid from silkworm pupas as the principal causative agent for the activity of the extract.

Pupas of silkworm (Bombyx mori Linné) were chosen here because extracts from these materials were reported to show high activity in the $\alpha$-amylase assay using rice endosperms. ${ }^{4}$ Fourteen kilograms of pupas was extracted according to the procedure shown in Fig. 1. Two hundred and thirty milligrams of active principle was obtained as pure crystals and indentified as fumaric acid on the basis of chemical and spectral evidences. Activity was assayed at each step of isolation and the acid thus obtained was found to be the sole agent responsible for the activity of the tissue.

The bioassay method employed here was

1) H. Yomo, Hakko Kyokai Shi, 18, 494 (1960); L. G. Paleg, Plant Physiol., 35, 293 (1960).

2) Y. Murakami, Bot. Mag. (Tokyo), 79, 315 (1966); Y. Ogawa, Plant Eु Cell Physiol., 7, 509 (1966); S. Mori, K. Kumazawa and S. Mitsui, ibid., 6, 571 (1965)

3) Y. Ogawa, Proc. Japan Acad., 41, 850 (1965).

4) Y. Ogawa and S. Imamura, Memoirs Coll. Agr. Kyoto Univ., No. 90, 27 (1966). almost identical with those used by Ogawa in his report, ${ }^{31}$ though some modifications were adopted for convenience. Rice seeds (Oryza sativa L. var. Koshijiwase) were dehusked and cut transeversely in half with a razor. The apical halves were dipped into $75 \%$ aqueous ethanol for $1 \mathrm{~min}$., washed with sterile distilled water and dipped into $10 \%$ aqueous antiformin for $10 \mathrm{~min}$. successively. Then the sterilized endosperms were washed several times with sterile distilled water.

The test sample was dissolved in $2 \mathrm{ml}$. of sodium acetate buffer $(0.01 \mathrm{~m}, \mathrm{pH} 4.8)$ in a test tube, which was then sterilized at $120^{\circ} \mathrm{C}$ for $5 \mathrm{~min}$. ${ }^{*}$ with a cotton stopper. Fifteen endosperms were transferred into each solution and incubated at $30^{\circ} \mathrm{C}$ with intermittent shaking at every $24 \mathrm{hrs}$. After $66 \mathrm{hrs}$. the endosperms were removed and total reducing sugars in the solution were measured according to the colorimetric method of Somogyi-Nelson.

Both natural and synthetic samples of fumaric acid were tested and found to be equally active in promoting sugar release. The minimal concentration of the acid necessary for activity was around 125 p. p. m. The stimulation was partially inhibited by the addition of 200 p. p. m. of chloramphenicol as shown in Fig. 2.

Several organic acids, i. e. stearic, oleic,

* It was confirmed beforehand that activity of the extract was unaffected through this process. 


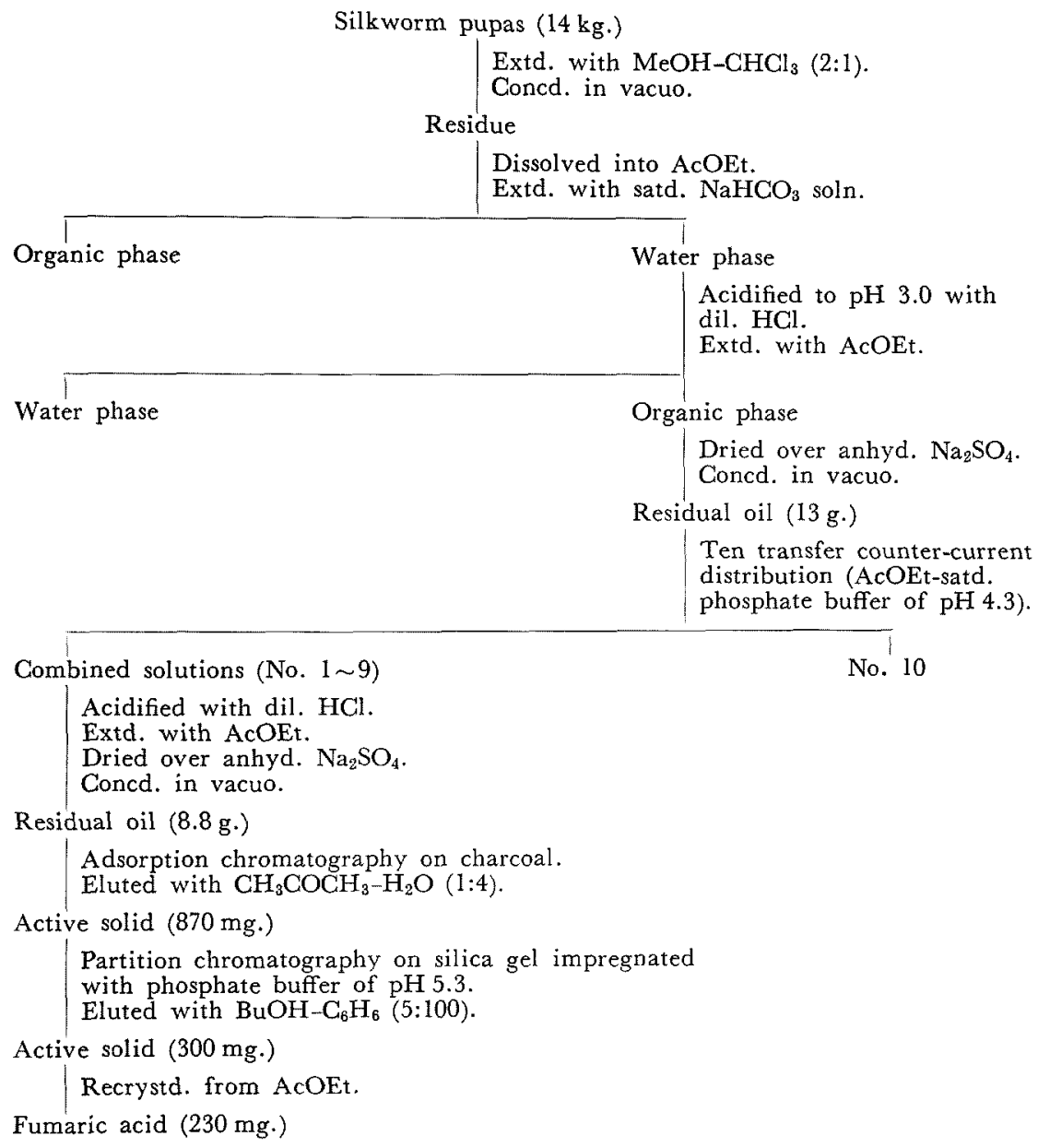

FIG. 1. Isolation Procedure of Fumaric Acid from Silkworm Pupas.

linoleic, linolenic, oxalic, succinic, malic and tartaric acids, were tested for activity in the assay. Oxalic and succinic acids were slightly active, their efficiencies at 250 p. p. m. being about one fourth of that of fumaric acid. These results were inconsistent, however, with the previous report ${ }^{5 \prime}$ that various organic acids including fumaric acid, which were adjusted to $\mathrm{pH} 5.5$ with $0.1 \mathrm{~m}$ sodium phosphate, were not active in the rice endosperm assay. Therefore, the efficacy of

5) Y. Ogawa, Plant $\mathcal{E}$ Cell Physiol., 7, 509 (1966). sodium fumarate was re-examined, and no activity in this assay was observed up to 500 p. p. m.

As shown in Table I, free fumaric acid in higher concentrations made test solutions acidic with only slight neutralization being observed after incubation. In the case of sodium fumarate, on the other hand, the $\mathrm{pH}$ value arose from 5.1 to 7.0 at 500 p. p.m. This raised the question as to whether the acidity of the solution had influenced sugar release from the endosperm. However, when the endosperm was incubated in $0.1 \mathrm{~m}$ phos- 


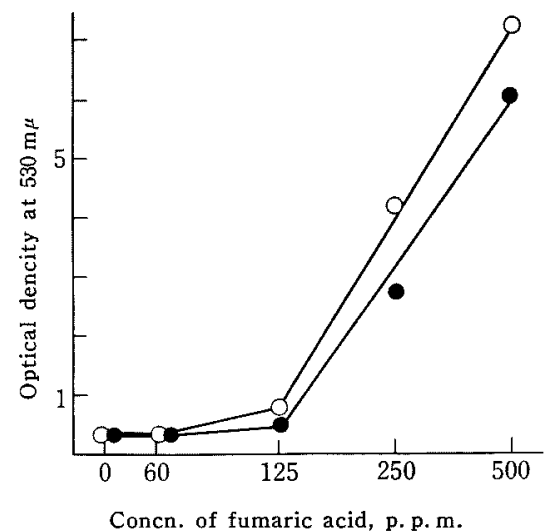

FIG. 2. Effect of Fumaric Acid on Reducing Sugar Release in Embryoless Rice Endosperms and Inhibitory Action of Chloramphenicol.

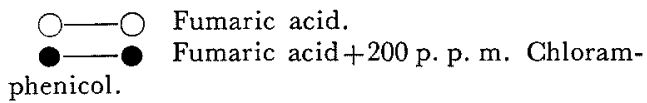

TABLE I. ACIDITy GHANGE OF MEDIUM DURING 66 HRS. INCUBATION AT $30^{\circ} \mathrm{C}$ IN THE PRESENCE OF FUMARIC ACID.

Concn. of fumaric acid p. p. m.

$\mathrm{pH}$ before incubation $\mathrm{pH}$ after incubation

$\begin{array}{lllll}500 & 250 & 125 & 60 & 0\end{array}$

$\begin{array}{llllll}3.6 & 3.8 & 4.3 & 4.8 & 4.8\end{array}$

$\begin{array}{lllll}4.2 & 4.6 & 5.0 & 5.6 & 6.2\end{array}$

phate buffer at $\mathrm{pH} 3.0$, promotion of sugar release was only one tenth of that incubated with 250 p. p. m. of fumaric acid. In addition, the filtrate from a homogenate of endosperms, which were incubated with 250 p. p. m. of fumaric acid for $66 \mathrm{hrs}$. and then separated from the solution, showed qualitatively far higher activity in the assay than that of the control incubated without the acid. This means that the effect of fumaric acid on the release of reducing sugars is due to an enzymatic hydrolysis and not to a direct acid hydrolysis by fumaric acid itself.

Thus the basis for the activity of silkworm pupa extracts in the embryoless rice endosperm assay has unequivocally clarified through the isolation of fumaric acid. Whether this acid promotes de novo synthesis of enzyme or release of bound enzyme should be the subject of further investigations. It is interesting that Murakami ${ }^{6 /}$ has recently found that such a toxic substance as chlorine increases reducing sugars due to the release of bound $\beta$-amylase in the rice endosperm assay.

We wish to express our thanks to Mr. K. Koizumi of Sericultural Experiment Station of Kanagawa Prefecture for supplying silkworm pupas. We are also grateful to Dr. Y. Murakami of National Institute of Agricultural Sciences, Dr. B. O. Phinney of University of California, Los Angeles and Dr. M. Katsumi of International Christian University for their valuable discussion.

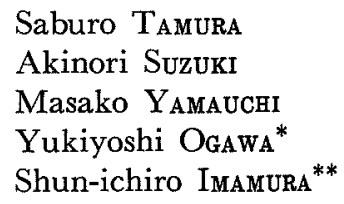

Received August 29, 1967

6) Y. Murakami, private communication. 